UVX 2010 (2011) 27-33

DOI: $10.1051 / \mathrm{uvx} / 2011004$

(C) Owned by the authors, published by EDP Sciences, 2011

\title{
Des états super-excités vers une empreinte Rydberg
}

\author{
V. Blanchet et R. Cireasa \\ Université de Toulouse, UPS, 118 route de Narbonne, 31062 Toulouse, France \\ CNRS, Laboratoire Collisions Agrégats Réactivité, IRSAMC, 31062 Toulouse, France
}

\begin{abstract}
Résumé. Les expériences pompe-sonde de photoionisation sont examinées dans le cas d'une ionisation à deux photons avec une résonance à un photon au voisinage du seuil d'ionisation. Les trois systèmes explorés sont $\mathrm{C}_{10} \mathrm{H}_{8}, \mathrm{CH}_{3} \mathrm{I}$ et $\mathrm{NO}_{2}$. De manière surprenante ces trois photoionisations présentent une empreinte Rydberg plus ou moins importante, soit une transition sonde vers un état doublement excité qui relaxe pendant la durée de l'impulsion sonde vers des états de Rydberg. Le second photon sonde photoionise alors ces états de Rydberg dans le cas de $\mathrm{C}_{10} \mathrm{H}_{8}$ et $\mathrm{CH}_{3} \mathrm{I}$ ou encore dissocie et photoionise dans le cas de $\mathrm{NO}_{2}$.
\end{abstract}

\section{INTRODUCTION}

Les réactions chimiques sont le résultat d'un changement de configuration électronique lors de l'approche entre réactifs. A l'échelle microscopique, ce changement de configuration induit des forces qui déplacent un ou plusieurs atomes à l'intérieur du système réactif. Résoudre temporellement la relaxation d'énergie initiée par l'absorption d'un photon est l'objectif de la femtochimie. Ceci est réalisé par des expériences pompe-sonde dans lesquelles deux techniques peuvent être différenciées : une sonde par absorption et une sonde par diffraction. La méthode émergeante de diffraction a pour principal avantage de révéler directement la position des noyaux sans aucune connaissance des surfaces de potentiel, complément nécessaire des techniques basées sur la spectroscopie. Ainsi en principe, toutes les demi-réactions chimiques peuvent être observées que ce soit une simple photodissociation ou plus complexe comme une photoisomérisation. Les nouvelles sources ultracourtes tel que le rayonnements $\mathrm{X}$ par laser électrons libres [1] ou encore plus prometteur les sources d'électrons [2] offrent en théorie la possibilité d'effectuer des expériences de diffraction résolues en temps. Toutefois, ces instruments sont au stade de la fiabilisation avec des points durs technologiques tels que la durée, le flux et la synchronisation temporelle de la sonde [3]. Les expériences alternatives les plus utilisées aujourd'hui sont basées sur la spectroscopie de photoionisation [4] enregistrée par imagerie de vecteur vitesse [5]. Rappelons que les premiers états électroniques de l'ion sont en effet les états excités les mieux caractérisés théoriquement et expérimentalement. L'imagerie, devenue l'outil de prédilection des expériences de femtochimie en phase gazeuse, est simple d'utilisation (quelque soit la polarité utilisée) avec une résolution en énergie de l'ordre des largeurs spectrales des impulsions femtosecondes malgré le volume de collection des $4 \pi$-stéradians. La dynamique est idéalement révélée à partir de la dépendance énergétique des photoélectrons émis en fonction du délai pompe-sonde sur espèces neutres [6], chargées [7] ou solvatées [8] ou encore celles des photofragments [9, 10]. Tout récemment, l'imagerie-femtoseconde de coïncidence photofragment/photoélectron a été développé $[11,12]$. Les potentiels d'ionisation étant généralement proches de la dizaine d'eV avec une énergie de pompe autour de 3 à $4 \mathrm{eV}$, la plupart des expériences pompe-sonde d'ionisation est souvent réalisée avec une sonde d'ionisation multiphotonique. C'est dans ce contexte que ce travail se place. Nous avons constaté sur quelques polyatomiques de petites tailles que pour une ionisation à deux photons impliquant une première transition proche du seuil d'ionisation, le spectre de photoélectrons était caractérisé

This is an Open Access article distributed under the terms of the Creative Commons Attribution-Noncommercial License 3.0, which permits unrestricted use, distribution, and reproduction in any noncommercial medium, provided the original work is properly cited. 
par une empreinte de plusieurs états de Rydberg. Nous présenterons dans ce qui suit le cas de la relaxation électronique de l'azulène $\left(\mathrm{C}_{10} \mathrm{H}_{8}\right)$, celui de la prédissociation de l'iodure de méthyle $\left(\mathrm{CH}_{3} \mathrm{I}\right)$ et finalement la dissociation du dioxide d'azote $\left(\mathrm{NO}_{2}\right)$. Ces systèmes et dynamiques sont tous différents et pourtant un même phénomène apparait, soit une empreinte Rydberg plus ou moins compétitive avec l'ionisation directe.

\section{MONTAGE EXPÉRIMENTAL}

Le montage est constituée d'une chaîne $\mathrm{kHz}$ femtoseconde amplifiée centrée à la longueur d'onde de $805 \mathrm{~nm}$ et accordable sur $15 \mathrm{~nm}$. La durée des impulsions, limitée par transformée de Fourier est variable entre 50 et 70 fs grâce à un filtrage spatial effectué par un modulateur acousto-optique (Dazzler) insérée avant amplification. L'accordabilité dans l'ultraviolet nécessaire à toute spectroscopie moléculaire est obtenue par un NOPA triple étages émettant dans le visible et compressé (nocollinear optical parametric amplification [13]). L'Ultraviolet (UV) est obtenue en sommant la sortie du NOPA avec le $805 \mathrm{~nm}$ dans des cristaux de beta barium borate (BBO) ou encore en générant la seconde harmonique du NOPA. Les impulsions sonde sont en général harmoniques du $805 \mathrm{~nm}$.Les énergies typiques utilisées sont de quelques $\mu \mathrm{J}$ pour l'impulsion pompe à quelques dizaines pour la sonde. La durée de corrélation croisée varie de $150 \mathrm{fs}$ à $300 \mathrm{fs}$ en fonction des longueurs d'onde utilisées. Ces impulsions sont combinées ensemble (angle $<5^{\circ}$ ) et focalisées indépendamment l'une de l'autre, sur un jet moléculaire continu de gaz neutre d'argon ensemencé des molécules étudiées dans une proportion de l'ordre de $10 \%$. Le spectre de photoélectrons, intégré sur typiquement $10^{5}$ tirs lasers, est enregistré par un imageur de vecteur vitesse. Cet imageur est constitué de trois lentilles électrostatiques projetant la particule chargée sur un détecteur bidimensionnel de galette à microcanaux couplé à un écran de phosphore (voir Figure 1). Les images collectées par une caméra CCD sont soustraites des fonds liés aux signaux par l'impulsion pompe seule et/ou l'impulsion sonde seule. Elles correspondent aux sphères de Newton intégrées le long de l'axe de temps de vol. Une coupe

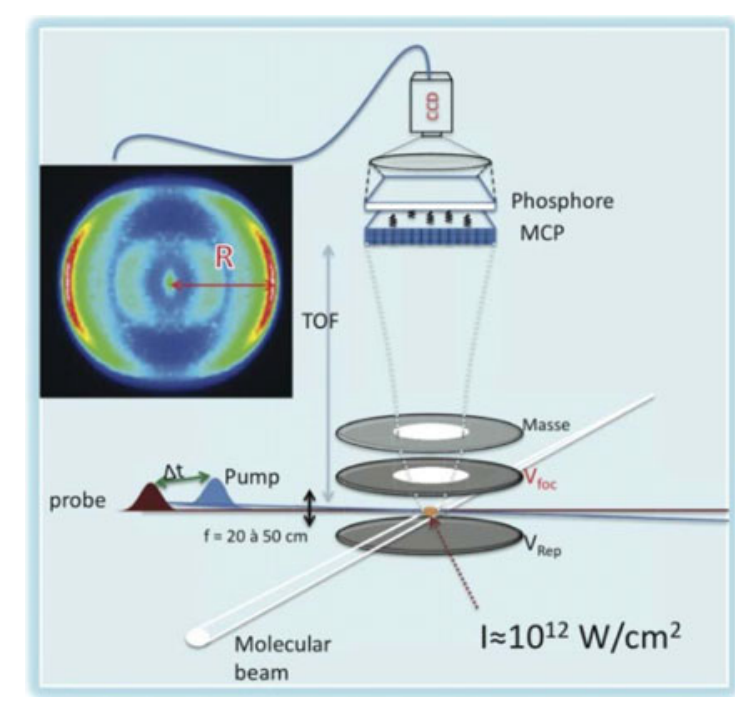

Figure 1. Principe de l'expérience avec l'énergie cinétique $E \propto R^{2}$. Un imageur est défini par le rapport $V_{\text {foc }} V_{\text {rep }}$ de 0.7 dans cette expérience. Ce rapport est plus ou moins maintenu à travers la gamme de tension de répulsion $V_{\text {rep }}$ quelque soit le signe de la polarité (positive pour des images d'ion et négative pour des images de photoélectrons). Notons que la tension maximale de $\mathrm{V}_{\text {rep }}$ est seulement imposée par les alimentations de haute tension ou encore les tensions de claquage. 
2D de cette distribution 3D est obtenue par une inversion d'Abel [14] sous condition d'une symétrie cylindrique autour d'un axe parallèle au plan du détecteur. Cette condition impose des polarisations de l'impulsion pompe et sonde toutes deux parallèles au détecteur. Ainsi le module et la direction des vecteurs vitesses peuvent être reconstitués, donnant alors la distribution angulaire et l'énergie cinétique des particules. L'énergie cinétique maximale résolue par imagerie, fixée par la valeur maximale de tension de répulsion $\left(\mathrm{V}_{\text {rep }}=6 \mathrm{kV}\right.$, Figure 1), la longueur du temps de vol $(42 \mathrm{~cm})$ et le diamètre du détecteur $(40 \mathrm{~mm})$, est de $\sim 4 \mathrm{eV}$ avec une résolution de $6 \%$. Le spectre de masse est obtenu simplement par la technique de temps de vol en collectant le courant de fuite de la dernière galette à microcanaux.

\section{RÉSULTATS}

Les longueurs d'ondes utilisées, les seuils d'ionisation, les énergies vibrationnelles des états peuplés par l'impulsion pompe et les durées de relaxation sont résumés dans le tableau 1.

Tableau 1. Schéma d'excitation. Le potentiel d'ionisation pour $\mathrm{NO}_{2}$ est celui de $\mathrm{NO}^{+}+\mathrm{O}$. L'énergie totale atteinte par l'absorption successive d'un photon pompe et d'un photon sonde $\left(1+1^{\prime}\right)$ est également indiquée.

\begin{tabular}{|c|c|c|c|}
\hline & $\mathrm{C}_{10} \mathrm{H}_{8}$ & $\mathrm{CH}_{3} \mathrm{I}$ & $\mathrm{NO}_{2}$ \\
\hline Pompe & 350 et $263 \mathrm{~nm} / 3.5 \mathrm{à} 4.7 \mathrm{eV}$ & $201.2 \mathrm{~nm} / 6.2 \mathrm{eV}$ & $269 \mathrm{~nm} / 4.6 \mathrm{eV}$ \\
\hline États & $\mathrm{S}_{2}$ & $\mathrm{~B}-6 \mathrm{~s}-\mathrm{E}_{3 / 2}$ & $\mathrm{~A}^{2} \mathrm{~B}_{2}$ \\
\hline Énergie vibrationnelle & 0 à $1.2 \mathrm{eV}$ & 0 & $3.35 \mathrm{eV}$ \\
\hline Temps de Relaxation & $30 \mathrm{ps} \mathrm{à} 3 \mathrm{~ns}$ & $1.3 \mathrm{ps}$ & $\sim 400 \mathrm{fs}$ \\
\hline sonde & $400 \mathrm{~nm} / 3.1 \mathrm{eV}$ & $400 \mathrm{~nm} / 3.1 \mathrm{eV}$ & $805 \mathrm{~nm} / 1.54 \mathrm{eV}$ \\
\hline$\left(1+1^{\prime}\right)$ & 6.6 à $7.8 \mathrm{eV}$ & 9.3 & 6.1 \\
\hline Potential d'ionisation (IP) & $7.4 \mathrm{eV}$ & $9.54 \mathrm{eV}$ & $12.38 \mathrm{eV}$ \\
\hline
\end{tabular}

Quelque soit le système considéré, l'ionisation requiert énergétiquement au moins l'absorption de deux photons sonde puisque $\left(1+1^{\prime}\right)<\mathrm{IP}$. Ceci reste vrai pour l'azulène excité à $4.7 \mathrm{eV}$ car l'état $\mathrm{S}_{2}$ peuplé par l'impulsion pompe a la propriété d'être strictement parallèle à l'état fondamental de l'ion. Ceci force une règle stricte d'ionisation directe en $\Delta v=0$ [15], et impose énergétiquement une ionisation à deux photons comme illustré sur la figure 2. Ainsi nous avons un schéma d'excitation totale de $\left(1+2^{\prime}\right)$.

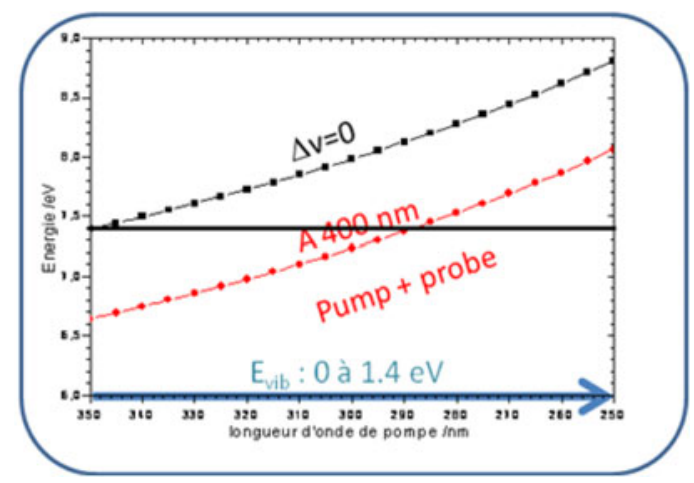

Figure 2. Energie totale $\left(1+1^{\prime}\right)$ en fonction de la longueur d'onde de la pompe pour $\lambda^{\prime}$ centré à $400 \mathrm{~nm}$ (courbe rouge). Le seuil d'ionisation à $7.4 \mathrm{eV}$ correspond à la droite horizontale tandis que l'ionisation verticale imposée par $\Delta \mathrm{v}=0$ correspond à la courbe noire, variant avec l'énergie vibrationnelle de l'état $\mathrm{S}_{2}$ indiquée en bleu. 
Nous démontrerons l'importance des états superexcités et des empreintes Rydberg sur l'azulène. La Figure 3-a présente les spectres de photoélectrons enregistrés en variant la longueur d'onde de la pompe et en gardant fixe la longueur d'onde de la sonde (voir Table 1). L'énergie totale $\left(1+2^{\prime}\right)$ varie par plus de $1.2 \mathrm{~V}$; pourtant la distribution énergétique des photoélectrons reste identique ne reflétant nullement le changement d'énergie totale ou encore le changement d'énergie vibrationnelle dans l'état $S_{2}$. Ceci est en total contradiction avec le fait qu'en général le spectre de photoélectron varie linéairement avec l'énergie totale disponible:

$$
\mathrm{E}_{\text {kin }}\left(\mathrm{e}^{-}\right)=\mathrm{E}_{\text {tot }}-\mathrm{E}_{\text {ion }}=\mathrm{h} v_{\text {pump }}+2 \mathrm{~h} v_{\text {probe }}-\mathrm{E}_{\text {ion }} .
$$

Les différent pics observés coïncident en fait à la spectroscopie connue des états de Rydberg de l'azulène [16-19], et ceci correspond à une empreinte Rydberg tel qu'introduit pour la première fois par P. Weber [20,21]. L'état $S_{2}$ étant parallèle à l'état fondamental de l'ion, il est également parallèle aux états de Rydberg qui par définition sont parallèles à l'état de l'ion. Ainsi la règle de transition $\Delta v=0$ s'impose aussi entre l'état $S_{2}$ et les états de Rydberg. S'il est possible de satisfaire à $400 \mathrm{~nm}$ cette règle pour un état de Rydberg, il est par contre impossible de la satisfaire pour tous les états de Rydberg observés sur la Figure 3-a. Ainsi un état relai doit intervenir dans cette transition à deux photons telle que résumé sur la Figure 3-c. Nous proposons que cet état relai soit un doublement excité (**) contenant avec un poids significatif, la contribution $\ldots\left(1 b_{1}\right)^{2}\left(2 b_{1}\right)^{2}\left(1 a_{2}\right)^{2}\left(3 b_{1}\right)^{2}\left(2 a_{2}\right)^{0}\left(4 b_{1}\right)^{0}\left(3 a_{2}\right)^{2}$. En effet cette configuration électronique est en bon
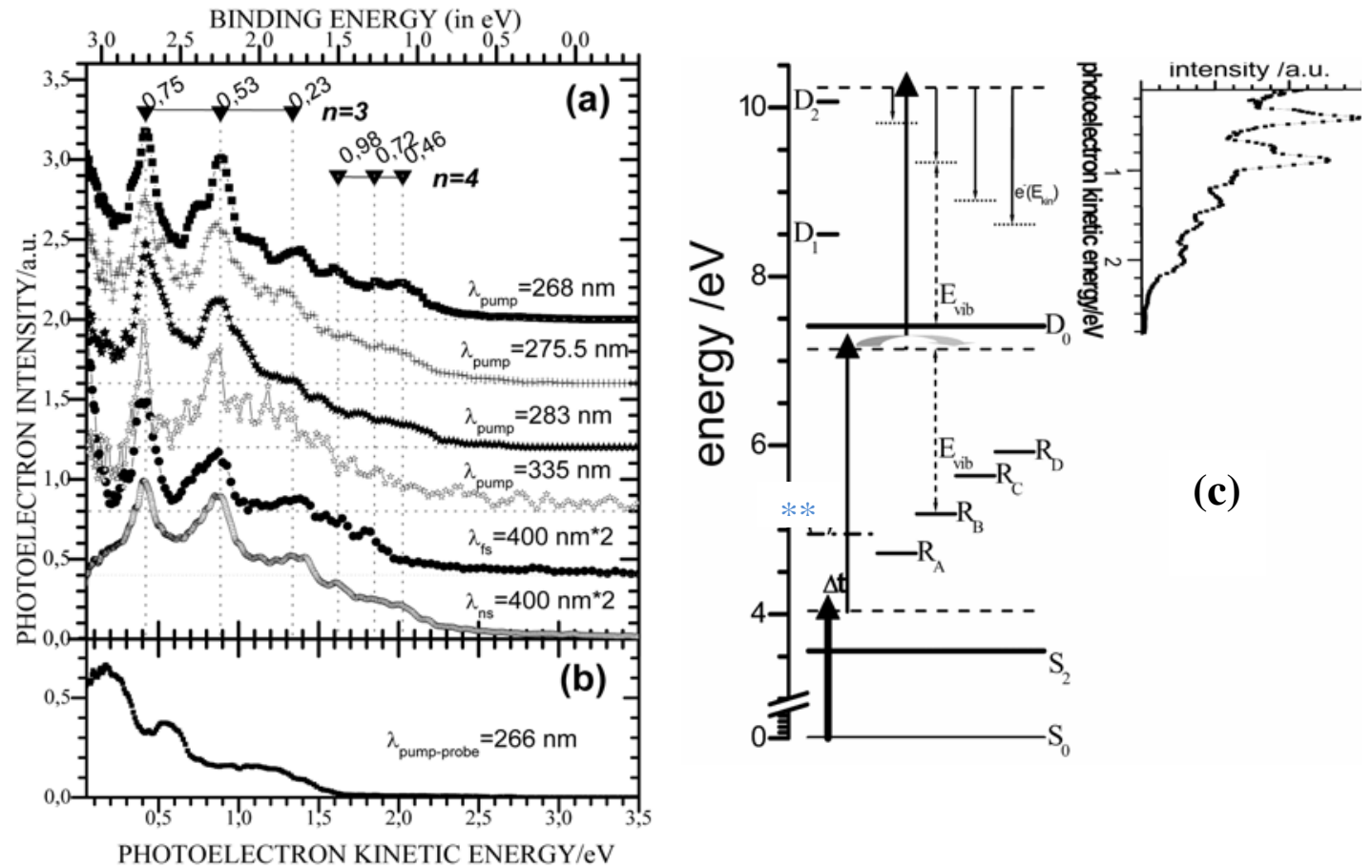

Figure 3. (a) Spectres de photoélectrons de l'azulène enregistrés en fonction de la longueur d'onde de la pompe, soit de l'énergie vibrationnelle dans $S_{2}$ pour une photoionisation prenant place à $400 \mathrm{~nm}$. Le spectre reste inchangé en fonction du délai pompe-sonde : seule une décroissance de l'intensité totale est observée, suivant la durée de vie de l'état $S_{2}$ [24]. L'énergie de liaison des états de Rydberg $\left(R_{A}, R_{B} \ldots\right.$ ) est indiquée sur l'axe supérieur ainsi que leurs nombres et défauts quantiques. (b) Un spectre de photoélectron obtenu avec une ionisation à $266 \mathrm{~nm}$ en $(1+1)$ ne présente aucune empreinte Rydberg. (c) Le schéma d'excitation par un doublement excité (**) avec empreinte Rydberg. Les états $\mathrm{D}_{i}$ sont les différents états électroniques de l'ion. 
accord avec un état de l'ion creux ... $\left(1 b_{1}\right)^{2}\left(2 b_{1}\right)^{2}\left(1 a_{2}\right)^{2}\left(3 b_{1}\right)^{2}\left(2 a_{2}\right)^{0}\left(4 b_{1}\right)^{0}\left(3 a_{2}\right)^{1}$ calculé très bas en énergie (à $9.6 \mathrm{eV}$ [22]) et un état doublement excité détecté par dichroïsme circulaire aussi bas que $4.95 \mathrm{eV}$ [23]. Ces états d'excitation creux ont en général une géométrie d'équilibre très différente des états simplement excités, permettant des transitions quelque soit l'énergie vibrationnelle.

Considérons un état de Rydberg d'énergie potentielle $R_{n}$ ou encore d'énergie de liaison $E_{b}^{n}=I P-R_{n}$. Après l'absorption d'un photon sonde on a (Figure 3-c):

$$
\hbar \omega_{\text {pump }}+\hbar \omega_{\text {probe }}=R_{n}+E_{\mathrm{vib}}^{R_{n}}
$$

$E_{\mathrm{vib}}^{\mathrm{R}}$ est l'énergie vibrationnelle dans l'état de Rydberg, énergie qui doit être conservée dans l'ion après absorption du second photon sonde $\left(E_{\mathrm{vib}}^{R_{n}} \approx E_{\mathrm{vib}}^{\mathrm{ion}}\right)$. Ainsi l'énergie cinétique du photoélectron s'écrit:

$$
E_{\text {kin }}^{e}=\left(\hbar \omega_{\text {pump }}+\hbar \omega_{\text {probe }}\right)+\hbar \omega_{\text {probe }}-E_{\mathrm{vib}}^{\mathrm{ion}}-I P=R_{n}+E_{\mathrm{vib}}^{\mathrm{ion}}+\hbar \omega_{\text {probe }}-E_{\mathrm{vib}}^{\mathrm{ion}}-I P
$$

Ou encore

$$
E_{\text {kin }}^{e}=\hbar \omega_{\text {probe }}+R_{n}-I P=\hbar \omega_{\text {probe }}-E_{b}^{n}
$$

Le spectre de photoélectron ne varie pas en fonction de l'énergie de la pompe; comme si l'énergie vibrationnelle avait été «effacée» du spectre de photoélectron. Cette invariance est reliée uniquement aux propriétés des états de Rydberg strictement parallèles à l'état de l'ion. Cette ionisation nous a permis de détecter l'anisotropie d'excitation de l'azulène [25].

(a)

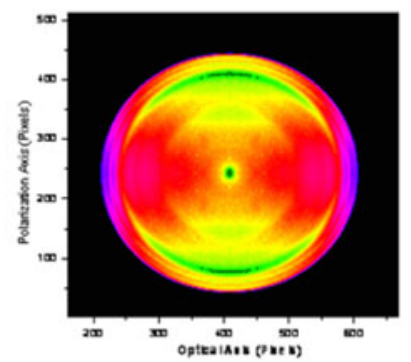

(b) $E_{3 / 2} \quad E_{1 / 2}$

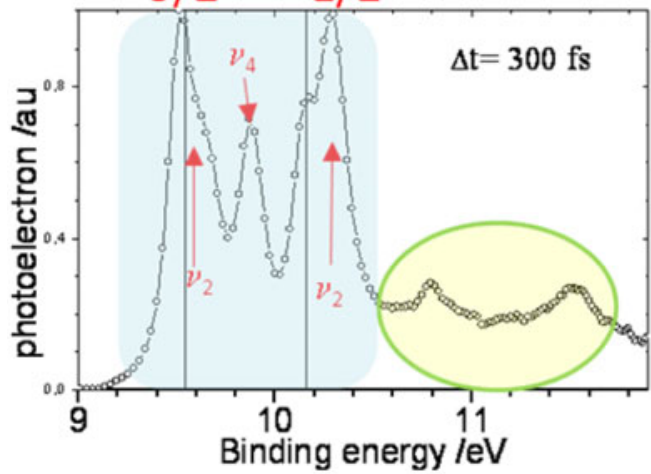

(c)

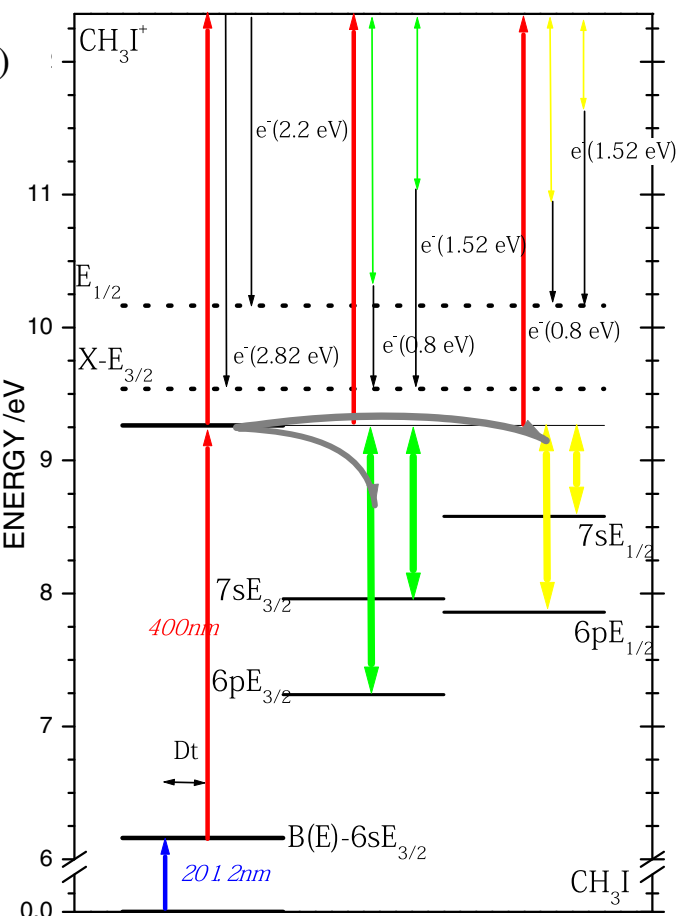

Figure 4. $\mathrm{CH}_{3} \mathrm{I}$ est excité dans le premier état de Rydberg $6 \mathrm{~s} \mathrm{E}_{3 / 2}$ puis ionisé tel que l'énergie totale est en $\left(1+2^{\prime}\right)$. (a) L'image et (b) la distribution de photoélectrons à 300 fs en fonction de l'énergie de liaison de l'ion restant. Les composantes en bleuté correspondent à l'ionisation directe tandis que l'ionisation avec empreinte Rydberg sont les composantes entourées à 10.8 et $11.5 \mathrm{eV}$. (c) Le schéma d'excitation avec l'empreinte Rydberg des états de 7s et $6 p$ des deux cœurs ioniques possibles $E_{1 / 2}$ et $E_{3 / 2}$ est en parfait accord avec la spectroscopie connue de ces états de Rydberg [27]. 


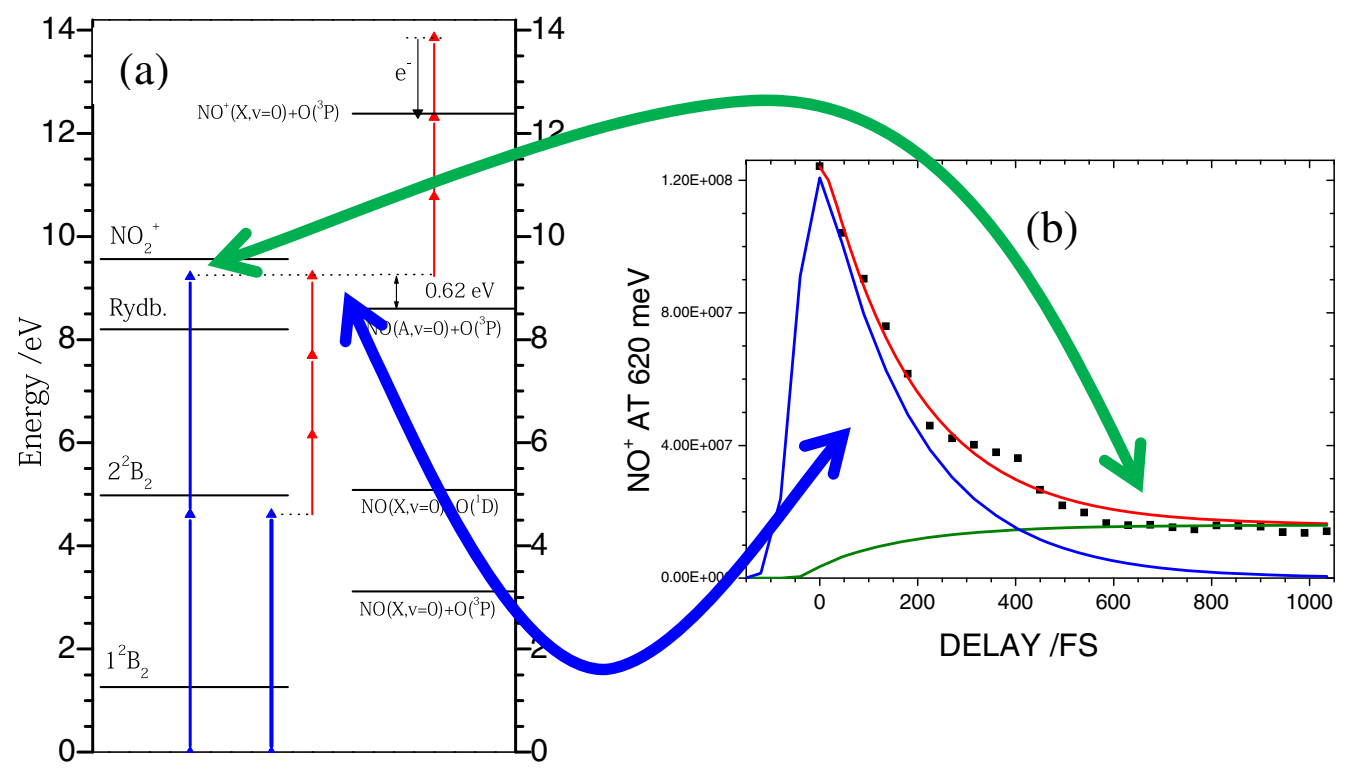

Figure 5. (a) Deux dynamiques de dissociation sont initiées dans $\mathrm{NO}_{2}$ : à un photon depuis 1 'état $1-^{2} \mathrm{~B}_{2}$ et à deux photons depuis les états de Rydberg [10]. Cette seconde dissociation est facile à détecter avec l'apparition suivant une loi exponentielle d'un fragment $\mathrm{NO}$ de $0.62 \mathrm{eV}$ d'énergie cinétique. La dépendance temporelle de cette composante est montrée en (b) et ne correspond en rien à une exponentielle croissante, mais plutôt à la somme d'une exponentielle décroissante (courbe bleu) avec une exponentielle croissante (courbe verte). En fait l'exponentielle décroissante correspond à la première dissociation détectée par empreinte Rydberg. Le bilan énergétique des fragments reste le même pour les deux dissociations du fait de l'harmonicité entre la pompe et la sonde.

La figure 4 illustre un résultat similaire observé sur $\mathrm{CH}_{3} \mathrm{I}$ [26] tandis que la figure 5 résume ce qui a été observé sur $\mathrm{NO}_{2}[10]$ avec les détails de longueur d'onde et d'énergie résumés dans la Table 1.

\section{CONCLUSIONS}

Dans ces trois exemples, les états de Rydberg sont peuplés à partir d'un état super-excité et pendant la durée même de la sonde sont photoionisés pour $\mathrm{CH}_{3} \mathrm{I}$ et $\mathrm{C}_{10} \mathrm{H}_{8}$ ou dissociés puis photoionisés dans le cas de $\mathrm{NO}_{2}$. Cette empreinte Rydberg est très compétitive dans l'azulène et reste anecdotique dans $\mathrm{CH}_{3} \mathrm{I}$. Ici, ces détections via des états de Rydberg n'apportent aucune sensibilité nouvelle à la dynamique, excepté dans $\mathrm{NO}_{2}$ pour qui cette dissociation à $266 \mathrm{~nm}$ était méconnue. Toutefois $\mathrm{P}$. Weber a démontré que les empreintes Rydberg étaient différentes d'un isomère de fluorophénols à l'autre [20] et ainsi cette sensibilité pouvaient être utilisée pour suivre des changements de conformation [28]. Cette détection assez simple à mettre en place techniquement semble très prometteuse pour suivre en temps réel des grands changements de structure sans pour autant être gêné par les variations inhérentes d'énergie vibrationnelle relié à ces changements. La limite de cette technique sera intrinsèquement reliée à la connaissance, même partielle, des états de Rydberg.

\section{Remerciements}

Les auteurs remercient N. Thiré (LCAR) et S. Pratt (Argonne) pour les resultats obtenus sur $\mathrm{CH}_{3} \mathrm{I}$ ainsi que B. Whitaker (Univ. Leeds) pour l'azulene. VB remercie le support technique du LCAR, notamment Laurent Polizzi pour les conceptions sous-vides, Stéphane Faure pour toute la partie interfaçage expérimentale et Elsa 
Baynard pour la maintenance de la chaîne laser. Ce travail est financé par l'ANR-COCOMUV et le Plan EtatRégion: Spectroscopies Optiques Ultimes' de la Région Midi-Pyrénées. RC remercie l'union Européenne pour le financement Intra-European Marie Curie (contrat MOLCOTUV-041732).

\section{Références}

[1] Barty, A., J. Phys. B 43 (2010) 194014.

[2] Zewail, A., H. , Science 328 (2010) 187-193.

[3] Ihee, H., et al., Int. Rev. Phys. Chem. 29 (2010) 453-520.

[4] Stolow, A., Annu. Rev. Phys. Chem. 54 (2003) 89-119.

[5] Eppink, A. T. J. B. and Parker, D. H., Rev. Sci. Instr. 68 (1997) 3477-3484.

[6] Suzuki, T., Annu. Rev. Phys. Chem. 57 (2006) 555-592.

[7] Surber, E. and Sanov, A., Phys. Rev. Lett. 90 (2003) 093001-093004.

[8] Sobhy, M. A., et al., The J. Phys. Chem. A 114 (2010) 11353-11363.

[9] Dura, J., et al., J. Chem. Phys. 131 (2009) 134311.

[10] Cireasa, R., et al., Phys. Scripta 80 (2009) 048106.

[11] Vredenborg, A., et al., J. Chem. Phys. 128 (2008) 204311.

[12] Rijs, A. M., et al., Phys. Rev. Lett. 92 (2004) 123002-123004.

[13] Cerullo, G., et al., Appl. Phys. Lett. 71 (1997) 3616-3618.

[14] Suzuki, T. and Whitaker, B. J., Int. Rev. Phys. Chem. 3 (2001) 313-356

[15] Weber, P. M. and Thantu, N., Chem. Phys. Lett. 197 (1992) 556-561.

[16] Kitagawa, T., et al., J. Mol. Spectr. 19 (1966) 1-3.

[17] Kuthirummal, N. and Weber, P. M., Chem. Phys. Lett. 378 (2003) 647-653.

[18] Kuthirummal, N. and Weber, P. M., J. Mol. Structure 787 (2006) 163-166.

[19] Lewis, J. W., et al., J. Phys. Chem. 87 (1983) 3611-3615.

[20] Gosselin, J. L. and Weber, P. M., J. of Phys. Chem. A Molecules (2005).

[21] Minitti, M. P., et al., J. Phys. Chem. A (2006).

[22] Deleuze, M. S., J. Chem. Phys. 116 (2002) 7012-7026.

[23] Gerhartz, W. and Michl, J., J. of Am. Chem. Soc. 100 (1978) 6877-6881.

[24] Blanchet, V., et al., J. Chem. Phys. 128 (2008) 164318.

[25] Raffael, K., et al., Chem. Phys. Lett. 460 (2008) 59-63.

[26] Thire, N., et al., Physical Chemistry Chemical Physics (2010).

[27] Dobber, M. R., et al., J. Chem. Phys. 99 (1993) 836-853.

[28] Minitti, M. P. and Weber, P. M., Phys. Rev. Lett. 98 (2007) 253004-253004. 